\title{
Capture and visualization of live Mycobacterium tuberculosis bacilli from tuberculosis bioaerosols
}

Ryan Dinkele, ${ }^{1,2 \dagger}$ Sophia Gessner, ${ }^{1,2 \dagger}$ Anastasia S. Koch, ${ }^{1,2}$ Carl Morrow, ${ }^{2,3}$ Melitta Gqada, ${ }^{3}$ Mireille Kamariza, ${ }^{4}$ Carolyn R. Bertozzi, ${ }^{5,6}$ Brian Smith, ${ }^{7}$ Courtney McLoud, ${ }^{7}$ Andrew Kamholz, Wayne Bryden, ${ }^{8}$ Charles Call, ${ }^{8}$ Valerie Mizrahi, ${ }^{1,2,9}$ Robin Wood, ${ }^{2,3 *}$ and Digby F. Warner, ${ }^{1,2,9^{*}}$

${ }^{1}$ SAMRC/NHLS/UCT Molecular Mycobacteriology Research Unit \& DST/NRF Centre of Excellence for Biomedical TB Research, Department of Pathology, Faculty of Health Sciences, University of Cape Town, South Africa.

${ }^{2}$ Institute of Infectious Disease and Molecular Medicine, Faculty of Health Sciences, University of Cape Town, South Africa.

${ }^{3}$ Desmond Tutu HIV Centre, University of Cape Town, South Africa.

${ }^{4}$ Department of Biology, Stanford University, CA 94305, USA.

${ }^{5}$ Department of Chemistry, Stanford University, CA 94305, USA.

${ }^{6}$ Howard Hughes Medical Institute, Stanford University, CA 94305, USA.

${ }^{7}$ Edge Embossing, USA

${ }^{8}$ Zeteo Tech, USA.

${ }^{9}$ Wellcome Centre for Infectious Disease Research in Africa, University of Cape Town, South Africa.

*To whom correspondence should be addressed: robin.wood@hiv-research.org.za; digby.warner@uct.ac.za

$\dagger$ These authors contributed equally to this work. 


\begin{abstract}
:
The tuberculosis (TB) pandemic demands urgent interventions such as those designed to interrupt Mycobacterium tuberculosis (Mtb) transmission, a challenge exacerbated by our poor understanding of the events enabling successful transfer of infectious bacilli between hosts. To address this problem, we developed the Respiratory Aerosol Sampling Chamber (RASC), a personal clean-room equipped with high-efficiency filtration and sampling technologies that allow biosafe capture and isolation of particulate matter - including Mtb bacilli - released by patients during natural breathing and (non-induced) cough. Here, we demonstrate the use of DMNtrehalose labelling to detect and quantify live $M t b$ bacilli among complex bioaerosol samples arrayed in a bespoke nanowell device following capture in the RASC. A pilot study identified $M t b$ in more than $85 \%$ of known TB patients, improving significantly on previous work which has relied on animal infection and cough sampling to estimate transmission events. Moreover, intrapatient comparisons of bioaerosol and sputum samples indicated that $M t b$ aerosols likely derive from a compartment other than sputum. These results support the utility of the RASC platform for research aimed at interrupting $M t b$ transmission, including the non-invasive detection of $M t b$ infected individuals who are predicted to contribute to bacillary spread despite the absence of clinical symptoms.
\end{abstract}

\title{
Introduction
}

Mycobacterium tuberculosis (Mtb) is the causative agent of tuberculosis (TB), the leading infectious killer globally having claimed 1.4 million lives in 2018 (1). TB control is almost entirely predicated on the treatment of active disease. However challenges posed by the long duration of standard chemotherapy $(2,3)$, delayed and missed diagnoses $(4,5)$, and failure to retain patients in treatment programmes, mean the goals of global TB eradication remain aspirational ( 6 ). The propagation of multi-drug resistant (MDR) disease further imperils this approach; an assessment reinforced by growing evidence of the primary transmission of MDR Mtb strains (7-12) and the knowledge that MDR-TB now accounts for more than one-quarter of all antimicrobial resistance (AMR) cases annually. These factors, together with the increasing appreciation that asymptomatic $M t b$ infections (with or without underlying pathology) might contribute to bacillary dissemination (13), have re-focused attention on $M t b$ transmission as a critical, but poorly understood, area for novel interventions in endemic regions (14-16). It is sobering, for example, that only $1-30 \%$ of new $M t b$ infections can be linked to known TB cases - a disconnect which implies the existence of many unrecognized transmitters in TB endemic communities (17-20).

An obligate pathogen, $M t b$ must drive successive cycles of transmission, infection, and disease in order to persist within the human population. Therefore, while the interval between host infection and disease can be variable (21-23) - perhaps involving extended periods of clinical latency in some cases (24-26) - the biological imperative to effect successful transmission (thus ameliorating the risks of extinction in an individual host) is inexorable and has been proposed to underlie the destructive lung pathology which enables aerosolization and release of $M t b$ bacilli $(21,27,28)$. Studying aerosolized $M t b$ is complicated by the low numbers of bacilli produced and the presence of environmental and patient-derived "contaminating" microorganisms and particulate matter (29). While molecular methods have enabled consistent and reliable detection of $M t b$ DNA in bioaerosols (30-32), these approaches cannot distinguish live from dead organisms nor do they allow investigations of the physiological state(s) of the aerosolized bacilli. The method of bacillary capture is also key: approaches based on forced or induced cough might not reflect natural transmission events $(33,34)$, whereas face-mask and related aerosol sampling methodologies either make live-cell analyses impossible (35) or require downstream in vitro propagation (via microbiological culture) (36), unavoidably altering the physiological and metabolic state of the captured samples.

In attempting to address these limitations, we developed a platform for the detection and capture of live Mtb bacilli from TB patients (37). Our Respiratory Aerosol Sampling Chamber (RASC) effectively functions as a personal clean-room, enabling collection and concentration of particulate material released by an individual patient during normal respiratory activity, including from natural (non-induced) cough (29,37). Through multiple iterations informed by our preliminary experimental and clinical observations, we have equipped the RASC to address systematically the comprehensive TB transmission research agenda outlined by others (38). Key questions posed include: When are viable, infection-competent bacilli released by $M t b$-infected individuals? How many aerosolized bacilli are produced, and are they single $M t b$ bacilli and/or clumps? Do clinically latent TB cases transmit $M t b$ ? What is the impact of anti-TB chemotherapy on production of viable Mtb aerosols? Does sputum smear-positivity predict infectiousness? Are sputum bacilli representative of the transmitted bacillary population? Is cough essential for transmission? Are 
some $M t b$ genotypes (better) adapted to transmission? How long do bacilli remain viable following aerosolization? Are some individuals "super-spreaders" with an increased propensity for Mtb bioaerosol production? To date, we have demonstrated the potential for liquid capture of aerosolized $M t b$ in the RASC, eliminating the dependency on solid culture-based techniques for bacillary detection $(29,37)$. This represents a key development, since it promises the possibility of detecting, isolating, and manipulating live bacilli for downstream phenotypic and genomic studies of transmission. For that, however, optimized protocols are needed to enable the specific labelling and concentration of low numbers of $M t b$ bacilli from mixed samples, and in a format amenable to detection via live-cell microscopy and, ultimately, experimental analysis.

The solvatochromic dye, 4- $N, N$-dimethylamino-1,8-napthalimide-trehalose (or DMN-tre), was developed as a fluorescent probe for the rapid detection of $M t b$ in sputum samples $(39,40)$. Enzymatic incorporation of this trehalose analogue into the hydrophobic mycomembrane by the mycobacterial Antigen-85 complex, as either a trehalose mono- or di-mycolate, enhances DMN fluorescence nearly one thousand-fold, limiting background noise attributable to unincorporated probe, and circumventing the need for multiple wash steps $(39,41)$. This is a key attribute of DMNtre and facilitates its use for the detection of $M t b$ bacilli within sputum in under an hour $(39,41)$. Importantly, since active membrane biosynthesis is a pre-requisite for bacterial labelling, DMNtre possesses the additional advantage of detecting live organisms only.

In this study, we combine the bioaerosol sampling capabilities of the RASC (37) with DMN-tre fluorescence detection (39) for the visualization and image-based phenotypic characterization of live $M t b$ bacilli in bioaerosol samples obtained from TB patients. We demonstrate that DMN-tre labelling of samples captured and concentrated in liquid enables fluorescence-dependent visualization of organisms in specialized nanowell arrayed devices. Then, in a pilot study, we use this system to detect putative $M t b$ in more than $85 \%$ of confirmed TB patients sampled, discerning three broadly distinct mycobacterial phenotypes associated with aerosolization. In addition, we compared matched bioaerosol and sputum samples to elucidate differences in labelling profiles. Our results establish the capacity for sensitive, non-invasive detection of infectious bioaerosols from TB-active individuals. Importantly, they also provide essential proof-of-concept data in support of current work which aims to extend sampling to asymptomatic, potentially $M t b$-positive individuals in addressing critical programmatic and scientific questions that must be tackled before interventions to interrupt TB transmission in endemic settings can be considered.

\section{Results}

Liquid cyclone capture, nanowell arraying and DMN-tre detection of TB bioaerosols

We previously described the capacity to enumerate $M t b$ genomes (via RD9-specific PCR) or $M t b$ colonies (via growth on solid medium loaded into different impactor devices) captured in bioaerosol material produced by tidal breathing and (non-induced) cough during the $\sim 60$ minute isolation of confirmed TB patients in the RASC (29,37). Although instructive, both methods (molecular and microbiological) are characterized by limitations which critically undermine their respective utilities for TB transmission research. On the one hand, PCR cannot differentiate live from dead bacilli, thereby rendering any estimate of viable $M t b$ release uncertain. On the other, culture-based methods are notoriously susceptible to contamination, the complications arising from the presence of so-called "viable but non-culturable" or "differentially detectable" organisms $(29,42)$, and the temporal - and, consequently, genetic (43) and physiological - separation of the (single) transmitted bacillus from the micro-population $\left(\sim 10^{6}\right.$ cells $)$ which eventually manifests as a visible $M t b$ colony on a plate. For these reasons, we deemed culture-independent detection, quantification and visualization of live organisms a priority.

Recent innovations in the RASC technology have ensured that approximately $500 \mathrm{~L}$ of expired air per subject per hour can be concentrated into $\sim 5-10 \mathrm{~mL}$ sterile phosphate-buffered saline (PBS). This development, which is enabled through the use of a Bertin Coriolis $\mu$ Biological Air Sampler, is significant in allowing high sampling volumes while producing a tractable, low-volume liquid output for downstream manipulation and analysis. Owing to its described utility for detecting $M t b$ in sputum samples from TB patients (39), DMN-tre was selected as the preferred label for the specific detection of $M t b$ within concentrated bioaerosol samples arrayed on a bespoke nanowell device (Figs. 1A \& 1B). The superstructure of each device consisted of two rows of eight wells (16 in total) overlaid on an embossed cyclic olefin copolymer (COC) film. The COC film contained the 50 × $50 \mu \mathrm{m}$ nanowells which were arrayed $\sim 140 \mu \mathrm{m}$ apart center-to-center (Fig. 1C). Each microwell therefore comprised approximately 1600 nanowells. 

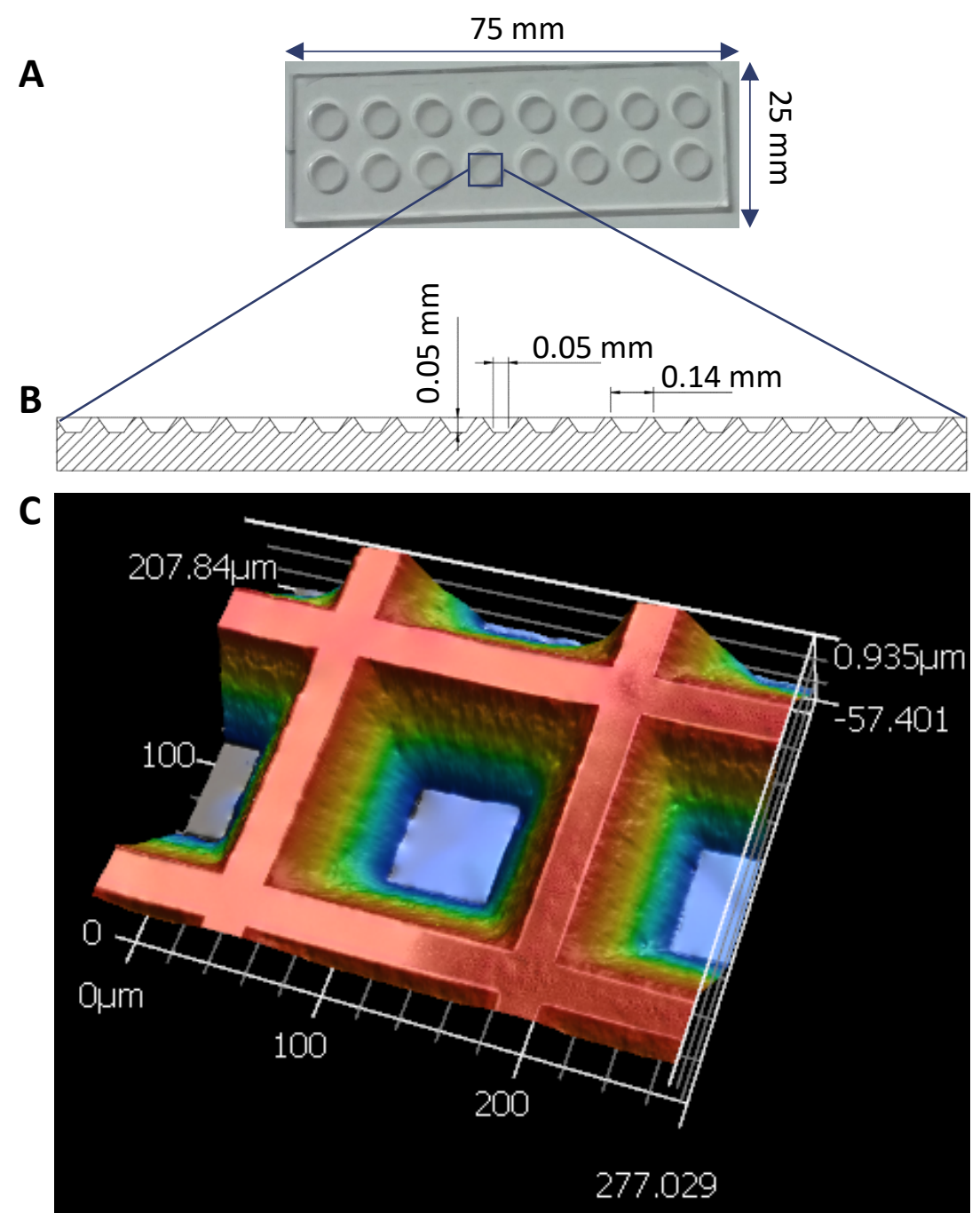

Fig. 1. Design and fabrication of nanowell-arrayed microscope slides for the compartmentalization and visualization of TB bioaerosols. (A) Photograph of a nanowell slide. (B) Schematic of the nanowell device. (C) 3D scan of a $207.840 \times 277.029$ $\mu \mathrm{m}$ section of the slide. Each device $(25 \times 75 \mathrm{~mm})$ consists of two rows of eight round microwells machined from cast acrylic. The microwells are $6 \mathrm{~mm}$ in diameter and $2 \mathrm{~mm}$ deep. The nanowell film, which is adhered to the superstructure via UV-curing adhesive, is made from embossed COC film. The nanowells have side-wall angles of $35^{\circ}$ and are 50 $\mu \mathrm{m}$ deep. The distance through the bottom of each well to the back of the film is roughly $170 \mu \mathrm{m}$, equivalent to a number 1.5 coverslip.

Following incubation in the presence of DMN-tre, the entire concentrated aerosol sample from a single patient was added to a single microwell. Thereafter, brief centrifugation of the device ensured dispersal of the liquid sample across thousands of individual nanowell compartments for fluorescence imaging. Physical separation of samples across the nanowells was considered a potential benefit in ensuring that all viable organic material was isolated in discrete wells, thereby facilitating detection while, potentially, reducing the likelihood that faster-growing non-Mtb organisms ("contaminants" for TB diagnostic purposes, but natural components of the patient aerosol microbiomes) would overwhelm the device following overnight DMN-tre labelling.

\section{Microscopic analyses revealed the complexity of bioaerosol samples}

To evaluate this technology, we initially recruited into a pilot study only TB-confirmed patients who had yielded GeneXpert-positive, drug-susceptible samples at presentation. Following routine diagnosis, the patients were transferred to the research site for RASC sampling; ethical approval sanctioned the delayed initiation of standard TB chemotherapy for 2 hours to enable the bioaerosol work. The $\sim 5-10 \mathrm{~mL}$ Bertin liquid-capture sample was concentrated via centrifugation before resuspension in $200 \mu \mathrm{l}$ standard Middlebrook $7 \mathrm{H} 9$ growth medium supplemented with $100 \mu \mathrm{M}$ DMN-tre for overnight $(16 \mathrm{~h})$ incubation at $37^{\circ} \mathrm{C}$ to allow metabolic incorporation (Fig. 2). 

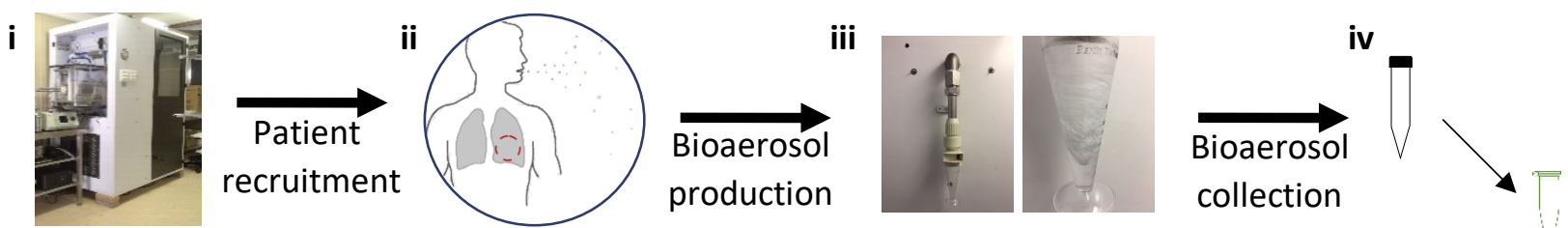

vii
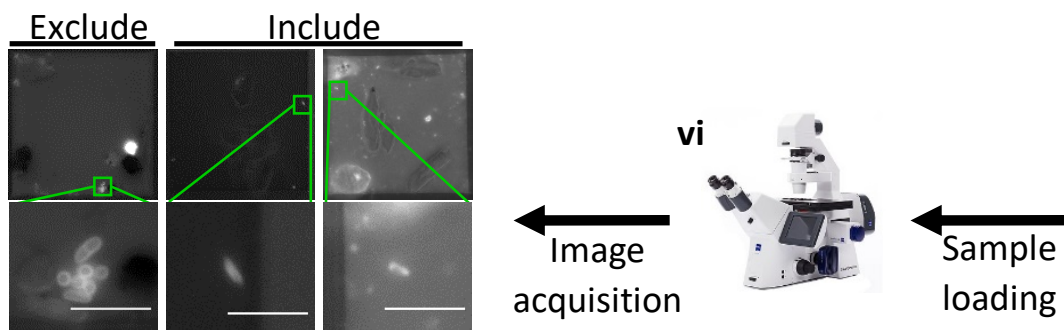

loading

Fig. 2. Workflow from participant recruitment to image analysis. (i) The Respiratory Aerosol Sampling Chamber (RASC). (ii) Bioaerosol production during tidal breathing and noninduced cough. (iii) Bioaerosol collection via Bertin Coriolis ${ }^{\mathrm{TM}} \mu$ Biological Air Sampler. (iv) Sample concentration and staining with $100 \mu \mathrm{M}$ DMN-tre during overnight ( $\sim 16$ hours) incubation at $37^{\circ} \mathrm{C}$. (v) The sample is arrayed in the nanowell device. (vi) Samples are scanned microscopically and bacilli counted. (vii) Relevant data are extracted from images. Scale bar, 5 $\mu \mathrm{m}$.

The bioaerosol samples of all participants contained large amounts of particulate matter (Fig. 3). A qualitative assessment indicated the presence of three broad categories of microparticles/debris: large microparticles with a crystalline appearance were the most common and possibly derived from the Tyvek suits that all participants were required to wear during sampling (Fig. 3A); small, auto-fluorescent "splotches", which were much less common (Fig. 3B); and, finally, nonfluorescent and non-staining "granular debris" which was also less common than the large crystalline debris and suggested the presence of human cellular material (Fig. 3C).
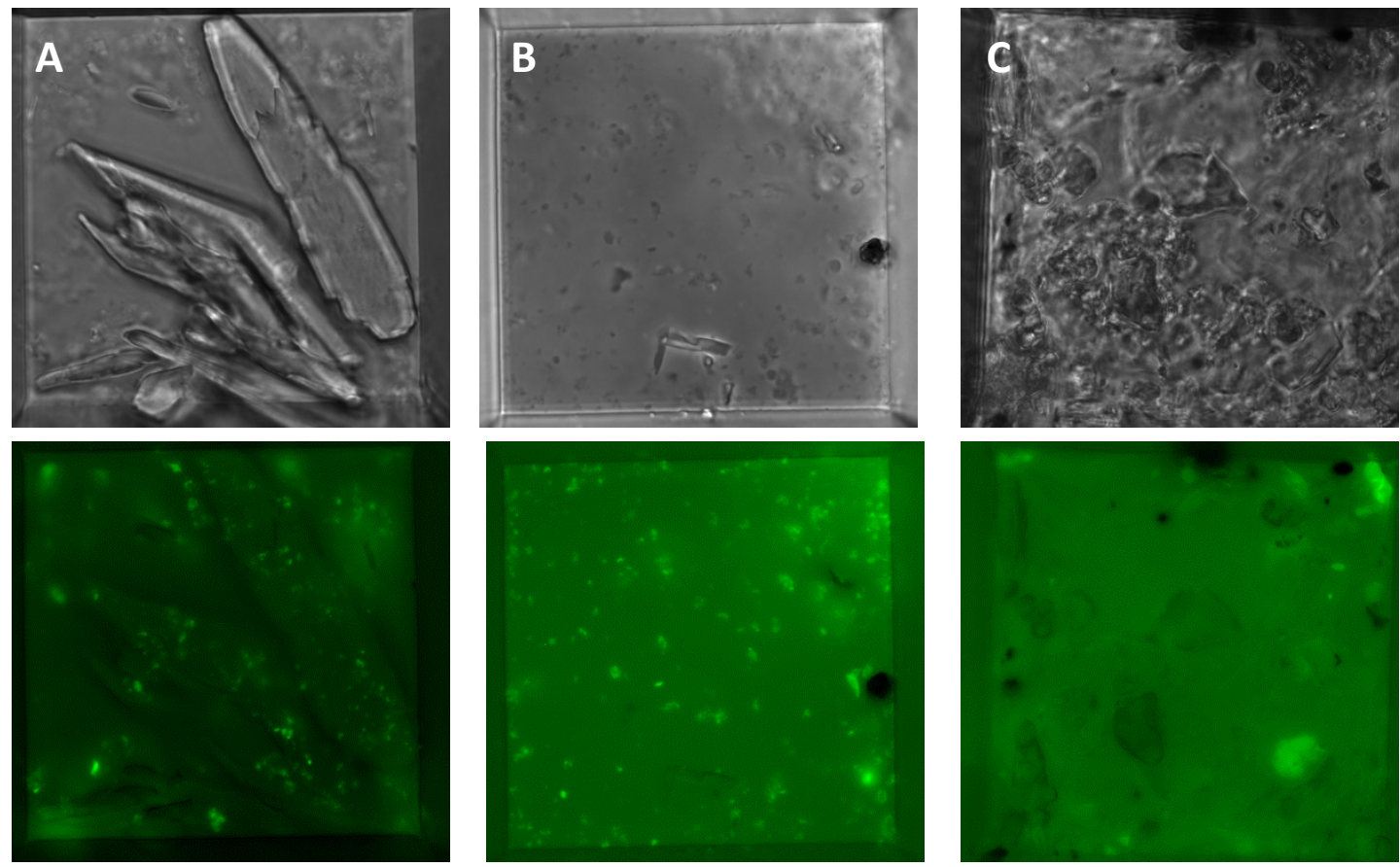

Fig. 3. Debris commonly found within TB bioaerosols. Representative images of the three major categories of debris found within bioaerosol samples after overnight staining with $100 \mu \mathrm{M}$ DMN-tre and visualization within a 50 × $50 \mu \mathrm{m}$ nanowell. (A) Large, crystalline debris, $(\mathbf{B})$ small fluorescent debris, and $(\mathbf{C})$ granular debris.

The presence of each of these categories of microparticle in any given sample was not mutually exclusive. Every patient produced large micro-particles; however, where the patient samples contained an additional type of debris, it was usually either granular debris or small, fluorescent debris. Further work is required to determine the precise composition and origin of the different 
micro-particles and, as noted below, to ascertain the relative contributions of auto-fluorescence versus true DMN-tre incorporation to some of the fluorescent structures.

\section{Microscopic investigation revealed the presence of non-Mtb DMN-tre organisms $^{+}$}

The physiological, metabolic and morphological states which characterize $M t b$ during bioaerosol release are not known but are critical to the visual detection of bacilli using the DMN-tre method. To provide a framework (or "identikit") for the assignment of "putative $M t b$ bacillus" to individual fluorescent structures/bodies detected in the nanowell device, we processed exponentially growing and aged cultures of the laboratory strain, $M t b$ H37Rv, via the clinical DMN-tre labelling algorithm (Fig. 4A). The median length of H37Rv bacilli did not change between exponential growth $(2.83 \mu \mathrm{m}, \mathrm{IQR}=0.85 \mu \mathrm{m})$ and early stationary phase $(2.77 \mu \mathrm{m}, \mathrm{IQR}=1.19 \mu \mathrm{m})(\mathbf{F i g} .4 \mathrm{~B})$. There was a small but reproducible change in average cell width, with bacilli entering early stationary phase $(0.60 \mu \mathrm{m}, \mathrm{IQR}=0.07 \mu \mathrm{m})$ very slightly thinner than exponentially growing organisms $(0.63, \mathrm{IQR}=0.04 \mu \mathrm{m})($ Fig. 4B). However, the average width for these two growth states overlapped substantially (Fig. 4B), leading us to reason that any organism falling outside this range $(\sim 0.47 \mu \mathrm{m}$ to $\sim 0.86 \mu \mathrm{m})$ should be disqualified from classification as "putative $M t b$ ".

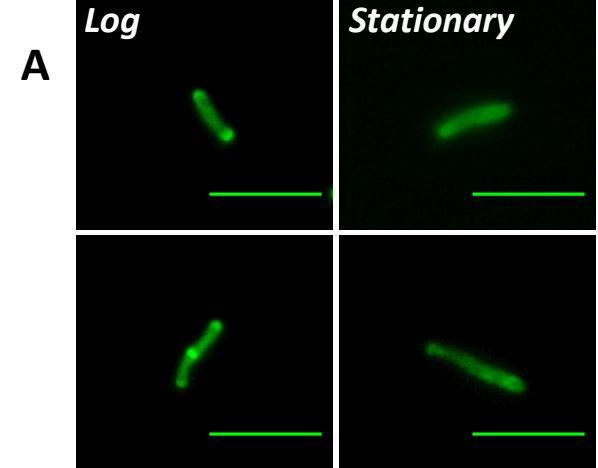

C Culture

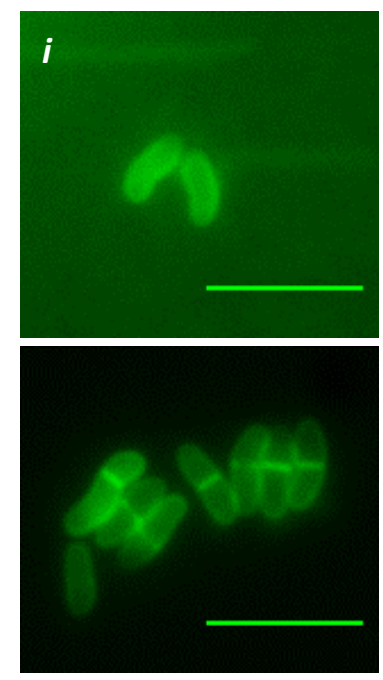

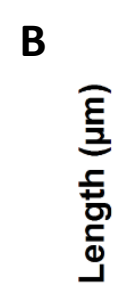

D

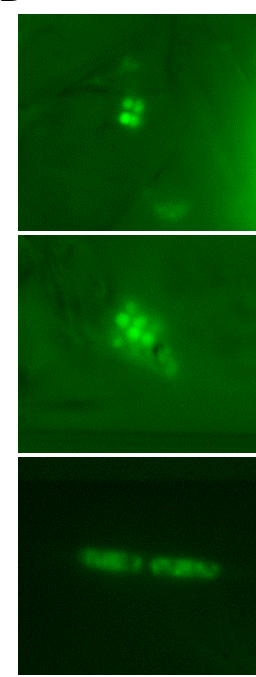

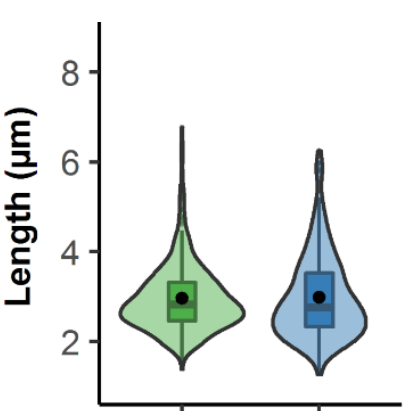

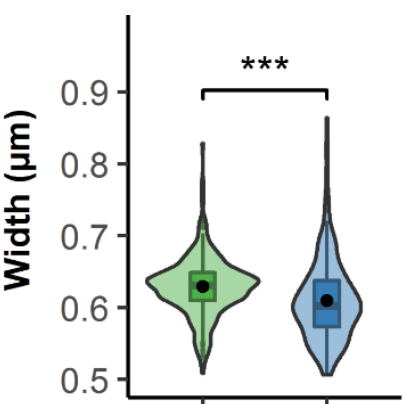

Log Phase $\quad$ Stationary Phase DMN-tre ${ }^{+}$organisms

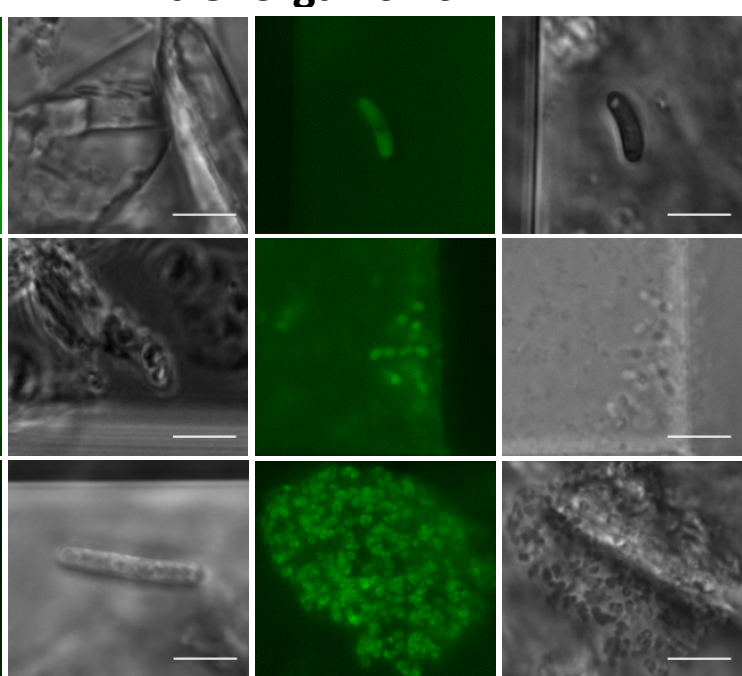

Fig. 4. Non-mycobacterial DMN-tre ${ }^{+}$organisms excluded owing to morphology and staining profile. (A) Representative images of $M t b \mathrm{H} 37 \mathrm{Rv}$ in log and stationary phase stained with DMNtre $(100 \mu \mathrm{M})$ for 2 hrs. (B) Plot comparing the length and width of $M t b$ in log-phase (green, $\mathrm{n}=347$ ) and stationary phase (blue, $\mathrm{n}=273$ ). $(\mathbf{C})$ Representative images of (i) C. striatum cultured in LB broth during log-phase and stained with $100 \mathrm{uM}$ DMN-tre for $5 \mathrm{~min}$, and (ii) DMN-tre positive organisms detected within a bioaerosol sample. (D) Corresponding fluorescent and phase contrast images of other non-Mtb, DMN-tre ${ }^{+}$organisms. Scale bar, $5 \mu \mathrm{m}$. Wilcoxon Rank-Sum test performed, $\mathrm{p}<0.001(* * *)$.

DMN-tre is not specific for $M t b$ : all bacteria within the Actinomycetales encoding homologs of the mycobacterial antigen 85 complex, which catalyzes trehalose mycolylation, possess the capacity to incorporate the fluorescent label. Among these, Corynebacteria are a common constituent of the oral microbiome and have previously been identified in TB sputum samples (39). To extend our database of potential DMN-tre-positive organisms, we incorporated the opportunistic pathogen, C. striatum (44-46), in the in vitro analyses. Following DMN-tre labelling via the established protocol, $C$. striatum was readily distinguishable from mycobacteria, yielding a distinct cytological (fluorescence incorporation) profile (Fig. 4C). Reassuringly, organisms with 
closely matching features were identified in clinical samples (Fig. 4C). However, we also observed multiple DMN-tre ${ }^{+}$organisms which might be of bacterial and/or fungal origin. Utilizing the exclusion criteria developed above, all of these organisms were eliminated from "putative Mtb" classification despite DMN-tre positivity (Fig. 4C \& D).

\section{Microscopic identification and characterization of DMN-tre ${ }^{+}$putative Mtb}

Applying the phenotypic classification system, we were able to identify putative $M t b$ in $89 \%$ $(25 / 28)$ of confirmed TB patients (Table 1). Our "empty booth" controls - expected to be Mtb free - returned $61 \%(14 / 23)$ positivity; however, the median count in the TB-positive patients was 15 (range 2-36), whereas the median count in the "positive" empty booths was 3 (range 1-10), suggesting the likelihood that the organisms detected represented carry-over from the cyclone collection system or RASC (Fig. 5A).

Table 1. Summary of patient characteristics

\begin{tabular}{|c|c|c|c|}
\hline & & n $(\%)$ & Median count $^{\dagger}$ \\
\hline \multirow[t]{3}{*}{ Sample type } & $\mathrm{TB}^{+}$patient & $28(54.9)$ & 15.0 \\
\hline & Empty RASC & $23(45.1)$ & 3.0 \\
\hline & Total & $51(100)$ & \\
\hline \multirow[t]{4}{*}{ Gender } & Male & $6(21.4)$ & 10.0 \\
\hline & Female & $7(25.0)$ & 7.0 \\
\hline & NA & $15(53.6)$ & 19.0 \\
\hline & Total & $28(100)$ & \\
\hline \multirow[t]{5}{*}{ HIV status } & Positive & $4(14.3)$ & 8.0 \\
\hline & Negative & $9(32.1)$ & 7.0 \\
\hline & NA & $15(53.6)$ & 19.0 \\
\hline & Total & $28(100)$ & \\
\hline & Yes & $7(25.0)$ & 7.0 \\
\hline \multirow[t]{3}{*}{ Smoking Status } & No & $5(17.9)$ & 7.0 \\
\hline & NA & $16(57.1)$ & 19.0 \\
\hline & Total & $28(100)$ & \\
\hline \multirow[t]{2}{*}{ Age } & Median $^{\dagger \dagger}$ & 32.5 & \\
\hline & IQR & 14.25 & \\
\hline
\end{tabular}

Three distinct labelling patterns were observed in the putative Mtb bacilli that were qualitatively comparable to incorporation patterns previously observed under various culture conditions (Fig. 5B). These were: a polar labelling pattern (Fig. 5B, panel i), a diffuse labelling pattern (Fig. 5B, panel ii), and a labelling pattern which resembled that seen previously in PBS-starved Mtb H37Rv in vitro (Fig. 5B, panel iii). It was notable that polar labelling of bacilli was only observed in patients producing small (auto)fluorescent debris, however the significance (and generalizability) of this observation remains to be determined. In addition, clumps and small clusters of organisms were detected in some samples (Fig. 5C). Further characterization of the bioaerosol was possible with single cell resolution. For example, the median cell length was compared for all participant samples in which $M t b$ was detected. These bacilli were on average shorter than in log-phase culture (Fig. 5D); in addition, the median length of bacilli found using the liquid sampling method described here was slightly shorter than that observed previously using the Andersen Impactor (29).

\section{Comparison of RASC and sputum profiles}

Sputum smear positivity has been a mainstay indicator of patient infectiousness $(47,48)$. Moreover, the accessibility and tractability of sputum has enabled numerous investigations of the physiology of infecting bacilli, the key assumption being that the sputum population provides a direct proxy of intrapulmonary and/or transmitted (aerosolized) Mtb organisms. To test this notion, we determined whether our sample concentration and DMN-tre labelling process could be applied in parallel to sputum and aerosol populations from the same patient. 

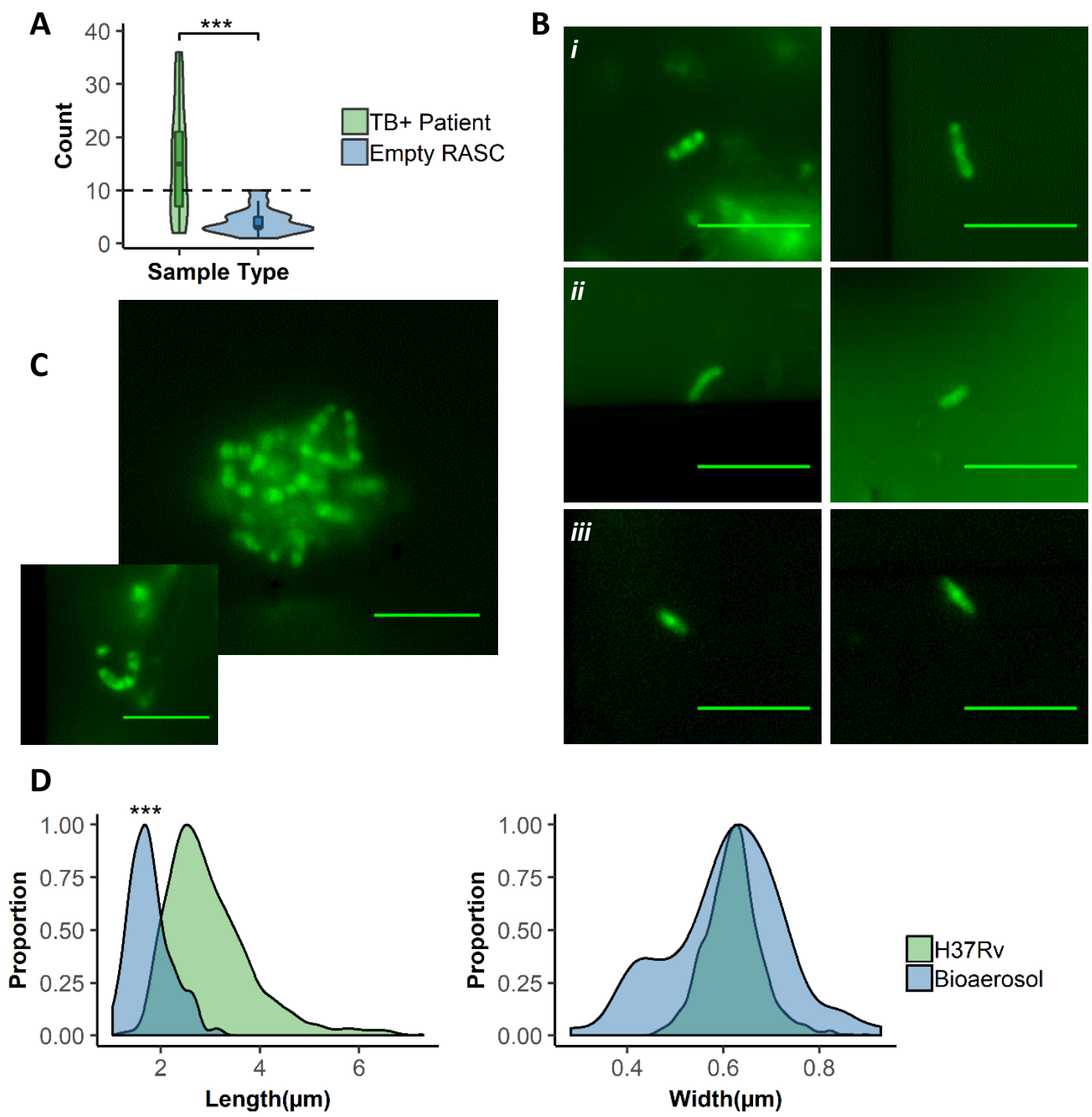

Fig. 5. Detection and characterization of putative $M t b$ within bioaerosols of confirmed

TB patients. (A) Plot comparing the number of putative $M t b$ detected within $\mathrm{TB}^{+}$participants (green, $\mathrm{n}=25 / 28(89.3 \%)$ ) and empty RASC controls (blue, $\mathrm{n}=14 / 23(60.9 \%)$ ). (B) Representative images of the three distinct cytological profiles (i-iii) observed in the 25 participants in which putative $M t b$ were detected. (C) Representative images of clumps of putative $M t b$ detected within bioaerosol samples. (D) Comparing distributions of cell lengths and widths in putative $M t b$ bacilli detected within bioaerosols of TB patients (blue) to Mtb H37Rv cultured within the lab (green). Scale bar, $5 \mu \mathrm{m}$. Wilcoxon Rank-Sum test performed, $\mathrm{p}<0.001(* * *)$.

An analysis of corresponding sputum and bioaerosol samples from 4 patients suggested a correlation in bacillary numbers between the two samples; however, the small sample size precluded assignment of statistical significance $(r=0.94, p=0.056)($ Fig. 6A). Notably, closer inspection of a single patient revealed that each sample was characterized by organisms displaying distinct labelling profiles (Fig. 6B). In addition, bacilli within the sputum sample tended to be shorter on average $(1.46 \mu \mathrm{m}, \mathrm{SD}=0.585 \mu \mathrm{m})$ than those found within the bioaerosol sample $(2.21$ $\mu \mathrm{m}, \mathrm{SD}=0.378 \mu \mathrm{m})($ Fig. 6B). Although limited to a single individual, this difference in length might derive from the collection method; that is, it could reflect the fact that aerosolized Mtb bacilli might experience a higher level of applied stress (e.g. torsional stress) during sample concentration via centrifugation, perhaps resulting in slight filamentation. Alternatively, the differences in staining and cell length might indicate that aerosolized $M t b$ bacilli do not necessarily originate from the sputum, but rather from the peripheral lung, a possibility reinforced by the recent observation that sputum bacillary load may not correlate with the size of the bacillary population captured in face-mask devices (33).

\section{Discussion}

The investigation of TB transmission spans approximately 140 years (49). However, until recently, technological limitations had mostly limited microscopic analyses to investigations of the sizes of 
droplet aerosols. Few attempts have been made to elucidate the physiological and metabolic states of aerosolized $M t b$ bacilli and how these might impact successful transmission to a new host. Similarly, the potential for other material in the aerosol - for example, host-derived cellular and molecular debris, and/or other "contaminating" microorganisms (the "transmission microbiome") - to influence transmission success is unknown.
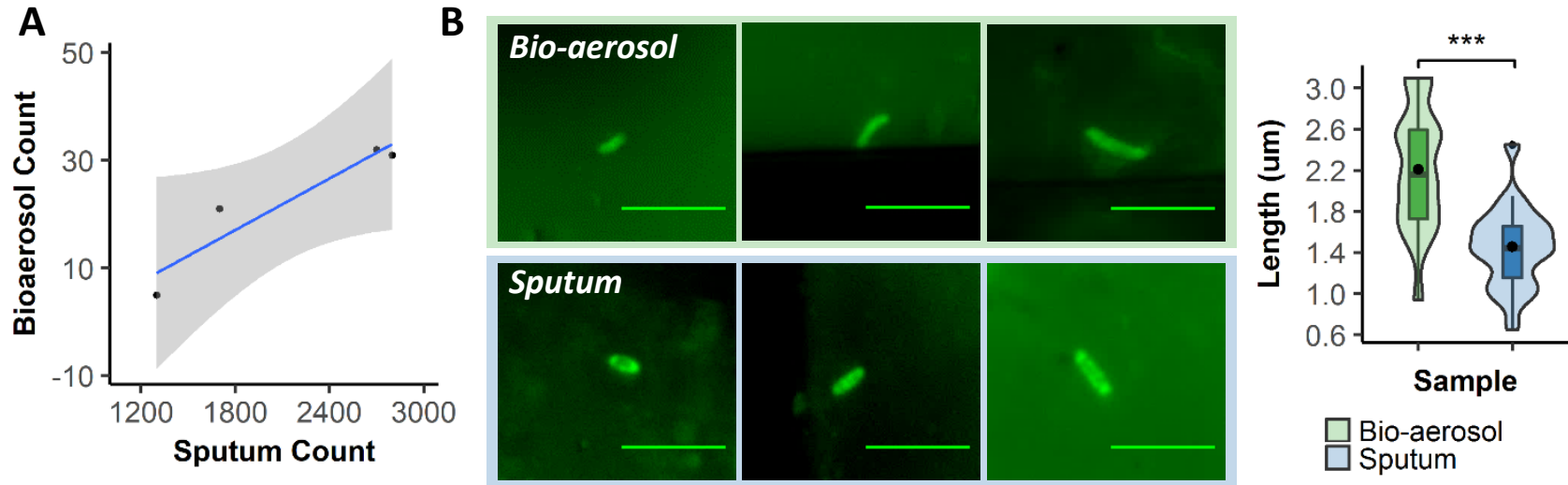

Fig. 6. DMN-tre staining and visualisation on the nanowell arrayed slides allows for direct, intra-patient comparison of bioaerosol and sputum samples. (A) An intra-patient comparison $(\mathrm{n}=4)$ of the number of putative $M t b$ identified within the bioaerosol compared to sputum $(r=0.94, p=0.056)$. (B) Representative images from a single patient's bioaerosol and sputum samples with a comparison of the length between putative Mtb bacilli isolated from bioaerosol (green, $\mathrm{n}=9$ ) and sputum (blue, $\mathrm{n}=38$ ) samples. Scale bar, $5 \mu \mathrm{m}$. Wilcoxon Rank-Sum test performed, $\mathrm{p}<0.001(* * *)$.

Our pipeline for visualization of bioaerosol samples derived from confirmed TB patients has allowed capturing and detection of live putative $M t b$ bacilli in $89 \%$ of GeneXpert-confirmed TB patients. From our microscopic analysis, it is evident that the composition of TB bioaerosols is complex and heterogeneous. In designing and implementing the RASC platform, considerable effort was made to minimize the collection of debris (for example, working in a clean-room and requiring that all patients wear Tyvek suits during the sampling process to reduce the production of non-aerosol-derived particulate matter), especially since the cleanliness of samples is critical for reliable microscopic visualization: auto-fluorescence represents a major confounder in environmental samples and there is also the possibility that debris might obscure Mtb bacilli partially or even fully. It was for this reason that the nanowell device was utilized, the intention being to maximise $M t b$ detection by increasing the likelihood of separating debris and bacilli through sample dispersal across thousands of nanowell chambers. Of course, it is likely that the composition and origin of the particulate matter might in future prove critical in determining precisely the anatomical origin of aerosolized bacilli. For now, though, raising the signal from putative $M t b$ above the background noise is our priority.

The chemical properties of DMN-tre, most notably the amplification of the fluorescence signal consequent on incorporation of the label into the mycomembrane, ensures its utility in detecting $M t b$ within decontaminated sputum (39). However, the fact that Ag85-dependent incorporation of trehalose into the mycomembrane is not unique to mycobacteria (39) complicates the application of this label on its own to sign confidently a classification of "Mtb positive" bacilli to aerosol samples. At the time this work was initiated, there was a lack of ancillary Mycobacterium-specific probes which could be applied to increase the specificity of the microscopic detection while retaining sample viability (thus excluding standard approaches including auramine and acid-fast staining which involve inactivation steps). More recently, other reagents have become available and are under investigation in the RASC platform, including (i) the CDG-DNB3 dual fluorescence probe which is activated by the mycobacterial BlaC $\beta$-lactamase and retained in the cell wall following covalent modification by the decaprenylphosphoryl- $\beta$-D-ribose 2 '-epimerase, DprE1 (50); (ii) the TAMRA-labelled benziothiazinone analogue, JN108, which also targets the mycobacterial membrane protein, DprE1, and according to its developers, is able to differentiate Corynebacterium from Mycobacterium on the basis of fluorescence localization (51); and (iii) the Quencher-Trehalose-Fluorophore (QTF) which, like DMN-tre, is a fluorogenic analogue of the natural substrate of mycobacterial mycolyltransferases (52). It is too soon to ascertain whether any of these will add value to the analysis; moreover, the overlapping auto-fluorescent signal in many samples urges the development of alternative probes in the red end of the visible spectrum.

The paucibacillary nature of the aerosol samples imposes additional complications which are not applicable to sputum. For example, the processing of bioaerosol samples does not include a 
decontamination step (sample loss is too significant a factor when dealing with tens of organisms) and, consequently, it is likely that proportionally larger numbers of non-Mtb DMN-tre-positive organisms are present in the bioaerosol samples than in decontaminated sputum. For this reason, ongoing work aims to refine our laboratory-based framework for identification of $M t b$ based on metabolic and morphological characteristics. Despite these limitations, we were able to identify with some confidence live putative $M t b$ in $89 \%$ of confirmed TB patients in our study. The detection of putative $M t b$ in "empty booth" controls was most likely due to the carry-over from the cyclone collection system or RASC. Current improvements to the collection system aim to facilitate complete sterilization and elimination of carry-over.

Research over the last 140 years has focused on symptomatic TB diseased individuals as the sole source of infectious particles (49). Every year, however, there are more than 10 million new TB cases globally with ongoing $M t b$ transmission the primary driver of incident disease. Despite significant advances in TB diagnostics, immunology and genomic epidemiology (53), much remains unknown about individual- and population-level transmission dynamics. This is because our current tools allow for the study of Mtb transmission only after a TB case is diagnosed. "Community-based" exposure studies focus predominantly on household contacts, which account for less than $20 \%$ of infections in high TB-burden settings (54-56). Moreover, the prolonged infectious period of TB (57-59) and the potential for transmission from brief, casual exposures mean that fewer than one-third of active TB cases can be epidemiologically and genetically linked $(17,19,20,46,54,60)$. In current work, we are adapting the RASC platform to identify viable aerosol production in subclinical cases (61), a key departure from prior research which has targeted $M t b$ detection almost exclusively to smear-positive TB.

\section{Materials and Methods}

\section{Ethics and Patient recruitment}

Ethical approval for this study was obtained from the Human Research Ethics Committee at the University of Cape Town (HREC 529/2019). Patients were recruited from two primary healthcare facilities in Masiphumelele and Ocean View, peri-urban townships located very close to each other outside Cape Town, South Africa. Informed consent was obtained from all participants and criteria for inclusion were (i) being 18 years or older, (ii) confirmed GeneXpert positive TB disease, and (iii) no evidence of drug resistant TB. All participants were recruited prior to initiation of standard anti-TB chemotherapy which commenced on the day of diagnosis after sampling was completed.

\section{Sample collection}

Participants recruited into this study were asked to provide two samples: a bioaerosol sample which was collected in the RASC (37) and a sputum sample. Bioaerosol collection was done according to previously described protocols (29). The sampling method was improved by using a Coriolis $\mu$ Biological Air Sampler (Bertin Technologies SAS, France) with the capacity to capture up to $500 \mathrm{~L}$ of expired air. Sputum samples were collected in the sputum booth at the clinic.

\section{Bacterial culture conditions and DMN-tre staining}

Culturing of all Mtb isolates was conducted in a Biosafety Level 3 (BSL3) facility. Mtb H37Rv was grown in Middlebrook $7 \mathrm{H} 9$ (Difco ${ }^{\mathrm{TM}}$ ) liquid broth supplemented with $0.2 \%$ (v/v) glycerol, $10 \%(\mathrm{v} / \mathrm{v})$ Middlebrook OADC enrichment and $0.05 \%$ (w/v) Tween 80 (Sigma-Aldrich) at $37^{\circ} \mathrm{C}$. Corynebacterium striatum was cultured in LB broth (Sigma-Aldrich) at $37^{\circ} \mathrm{C}$. For PBS starvation an H37Rv culture was grown to log-phase after which a 1:100 dilution was prepared in fresh $7 \mathrm{H} 9$. The diluted cells were harvested by centrifugation $(3000 \mathrm{xg}$ for $10 \mathrm{~min}$ ) and washed three times with an equal amount to original volume PBS $+0.05 \%$ tyloxapol. Following the final wash, the $\mathrm{OD}_{600}$ was adjusted to $\sim 1$ and incubated at $37^{\circ} \mathrm{C}$ for 14 days. For staining of exponentially replicating and stationary-phase bacilli, $M t b \mathrm{H} 37 \mathrm{Rv}$ was grown to an $\mathrm{OD}_{600} \sim 0.5$ and $\sim 1.2$, respectively. Bacteria were stained with $100 \mu \mathrm{M}$ DMN-trehalose for $2 \mathrm{~h}$. Stained cells were harvested by centrifugation at $13000 \mathrm{x} \mathrm{g}$ for $5 \mathrm{~min}$ before resuspending in PBS prior to visualization.

\section{Staining of samples for visualization in the nanowell device}

Staining of all clinical samples was conducted in a Biosafety Level 3 (BSL3) facility.

\section{1) Bioaerosol samples}

The 5-10 mL bioaerosol samples were concentrated by spinning at $3000 \mathrm{x} g$ for $10 \mathrm{~min}$ (Allegra ${ }^{\circledR}$ $\mathrm{X}-15 \mathrm{R}$, Beckman Coulter). The pellet was resuspended in $200 \mu \mathrm{l}$ fresh $7 \mathrm{H} 9$ and stained overnight $(12-16 \mathrm{~h})$, following which the stained sample was concentrated at $13000 \mathrm{x} \mathrm{g}$ for $5 \mathrm{~min}$ and resuspended in $20 \mu \mathrm{l}$ sterile, filtered PBS. 


\section{2) Sputum}

Sputum was treated with 2,3-dihydroxybutane-1,4-dithiol (DTT) (Sigma-Aldrich). An equal volume of $6.5 \mathrm{mM}$ DTT was added to the sputum sample and vortexed until liquified. The liquified sputum was centrifuged at $3000 \times \mathrm{g}$ for $15 \mathrm{~min}$ at $8^{\circ} \mathrm{C}$, after which the pellet was resuspended in PBS. To reduce any background host cell material, the MolYsis ${ }^{\mathrm{TM}}$ Complete5 kit (Molzym) was used according to manufacturer's instructions up to the point of bacterial lysis, resuspending the final pellet containing intact bacterial cells in $200 \mu 17 \mathrm{H} 9$. At this point, the suspension was stained as per bioaerosol staining described above.

Prior to any inoculation, the nanowell device (Edge Embossing) was plasma-coated (Novascan) to counteract the hydrophobicity caused by the polymer used to synthesise the nanowell plate. Samples were loaded into their respective wells and sealed using an adhesive film (ThermoFischer Scientific). The inoculated and sealed plates were centrifuged at $3000 \mathrm{x} g$ for $10 \mathrm{~min}$ after which they were removed from the BSL3 laboratory for visualization.

\section{Fluorescence microscopy}

Imaging was done on a Zeiss Axio Observer 7 equipped with a 100x plan-apochromatic phase 3 oil immersion objective with a numerical aperture of 1.4. Epifluorescent illumination was provided using a $475 \mathrm{~nm}$ LED and non-specific fluorescence was removed, where possible, with a Zeiss 38 HE filter set. Images were acquired using the Zeiss Zen software, and quantitative data were extracted using the MicrobeJ plugin for ImageJ (62).

\section{Statistical analysis}

Data were exported from ImageJ and all analysis was performed using R, version 3.5.1. Data normality was assessed visually and, where applicable, a Wilcoxon Rank-Sum test was performed.

\section{Supplementary Materials}

No supplementary materials are provided with this submission.

\section{References}

1. World Health Organization. Global Tuberculosis Report. (2019).

2. Johnson, J. L., Hadad, D. J., Dietze, R., Maciel, E. L. N., Sewali, B., Gitta, P., Okwera, A., Mugerwa, R. D., Alcaneses, M. R., Quelapio, M. I., Tupasi, T. E., Horter, L., Debanne, S. M., Eisenach, K. D., Boom, W. H. Shortening treatment in adults with noncavitary tuberculosis and 2-month culture conversion. Am. J. Respir. Crit. Care Med. 180, 558-563 (2009).

3. Wallis, R. S., Hafner, R. Advancing host-directed therapy for tuberculosis. Nat Rev Immunol 15, 255-263 (2015).

4. Naidoo, P., Theron, G., Rangaka, M. X., Chihota, V. N., Vaughan, L., Brey, Z. O., Pillay, Y. The South African Tuberculosis Care Cascade: Estimated Losses and Methodological Challenges. J. Infect. Dis. 216, S702-S713 (2017).

5. Kaplan, G. Tuberculosis control in crisis-causes and solutions. Prog. Biophys. Mol. Biol. 14 (2019).

6. Schito, M., Hanna, D., Zumla, A. Tuberculosis eradication versus control. Int. J. Infect. Dis. 56, 10-13 (2017).

7. De Vos, M., Müller, B., Borrell, S., Black, P. A., Van Helden, P. D., Warren, R. M., Gagneux, S., Victor, T. C. Putative compensatory mutations in the rpoc gene of rifampinresistant Mycobacterium tuberculosis are associated with ongoing transmission. Antimicrob. Agents Chemother. 57, 827-832 (2013).

8. Dheda, K., Limberis, J. D., Pietersen, E., Phelan, J., Esmail, A., Lesosky, M., Fennelly, K. P., te Riele, J., Mastrapa, B., Streicher, E. M., Dolby, T., Abdallah, A. M., Ben-Rached, F., Simpson, J., Smith, L., Gumbo, T., van Helden, P., Sirgel, F. A., McNerney, R., Theron, G., Pain, A., Clark, T. G., Warren, R. M. Outcomes, infectiousness, and transmission dynamics of patients with extensively drug-resistant tuberculosis and home-discharged patients with programmatically incurable tuberculosis: a prospective cohort study. Lancet Respir. Med. (2017). doi:10.1016/S2213-2600(16)30433-7

9. Gandhi, N. R., Moll, A., Sturm, A. W., Pawinski, R., Govender, T., Lalloo, U., Zeller, K., Andrews, J., Friedland, G. Extensively drug-resistant tuberculosis as a cause of death in patients co-infected with tuberculosis and HIV in a rural area of South Africa. Lancet 368, 1575-1580 (2006). 
10. Pietersen, E., Ignatius, E., Streicher, E. M., Mastrapa, B., Padanilam, X., Pooran, A., Badri, M., Lesosky, M., van Helden, P., Sirgel, F. A., Warren, R., Dheda, K. Long-term outcomes of patients with extensively drug-resistant tuberculosis in South Africa: A cohort study. Lancet 383, 1230-1239 (2014).

11. Wells, C. D., Cegielski, J. P., Nelson, L. J., Laserson, K. F., Holtz, T. H., Finlay, A., Castro, K. G., Weyer, K. HIV Infection and Multidrug-Resistant Tuberculosis-The Perfect Storm. J. Infect. Dis. 196, S86-S107 (2007).

12. Becerra, M. C., Huang, C. C., Lecca, L., Bayona, J., Contreras, C., Calderon, R., Yataco, R., Galea, J., Zhang, Z., Atwood, S., Cohen, T., Mitnick, C. D., Farmer, P., Murray, M. Resistance At No Cost: the Transmissibility and Potential for Disease Progression of DrugResistant M. Tuberculosis. bioRxiv 475764 (2018). doi:10.1101/475764

13. Xu, Y., Cancino-Muñoz, I., Torres-Puente, M., Villamayor, L. M., Borrás, R., BorrásMáñez, M., Bosque, M., Camarena, J. J., Colomer-Roig, E., Colomina, J., Escribano, I., Esparcia- Rodríguez, Gil-Brusola, A., Gimeno, C, Gimeno-Gascón, A, Gomila-Sard, B., González-Granda, D, Gozalo-Jiménez, N, Guna-Serrano, M., R, López-Hontangas, J. L., Martín-González, C., Moreno-Muñoz, R., Navarro, D., Navarro, M., Orta, N., Pérez, E., Prat, J., Rodríguez, J. C., Ruiz-García, M. M., Vanaclocha, H., Colijn, C., Comas, I. Highresolution mapping of tuberculosis transmission: Whole genome sequencing and phylogenetic modelling of a cohort from Valencia Region, Spain. PLoS Med. 16, e1002961 (2019).

14. Khan, P. Y., Yates, T. A., Osman, M., Warren, R. M., Heijden, Y. Van Der, Padayatchi, N., Nardell, E. A., Moore, D., Mathema, B., Gandhi, N., Eldholm, V., Dheda, K., Hesseling, A. C., Mizrahi, V., Rustomjee, R. Transmission of drug-resistant tuberculosis in HIV-endemic settings. Lancet Infect. Dis. 19, 1-25 (2019).

15. Peters, J. S., Andrews, J. R., Hatherill, M., Hermans, S., Martinez, L., Schurr, E., van der Heijden, Y., Wood, R., Rustomjee, R., Kana, B. D. Advances in the understanding of Mycobacterium tuberculosis transmission in HIV-endemic settings. Lancet Infect. Dis. 19, e65-e76 (2019).

16. Koch, A. \& Cox, H. Preventing drug-resistant tuberculosis transmission. Lancet Infect. Dis. (2019).

17. Crampin, A. C., Glynn, J. R., Traore, H., Yates, M. D., Mwaungulu, L., Mwenebabu, M., Chaguluka, S. D., Floyd, S, Drobniewski, F., Fine, P. E. M. Tuberculosis transmission attributable to close contacts and HIV status, Malawi. Emerg. Infect. Dis. 12, 729-735 (2006).

18. Glynn, J. R., Guerra-Assunção, J. A., Houben, R. M. G. J., Sichali, L., Mzembe, T., Mwaungulu, L. K., Mwaungulu, J. N., McNerney, R., Khan, P., Parkhill, J., Crampin, A. C., Clark, T. G. Whole genome sequencing shows a low proportion of tuberculosis disease is attributable to known close contacts in rural Malawi. PLoS One 10, 1-12 (2015).

19. Middelkoop, K., Mathema, B., Myer, L., Shashkina, E., Whitelaw, A., Kaplan, G., Kreiswirth, B., Wood, R., Bekker, L. G. Transmission of tuberculosis in a south African community with a high prevalence of HIV infection. J. Infect. Dis. 211, 53-61 (2015).

20. Verver, S., Warren, R. M., Munch, Z., Richardson, M., van der Spuy, G. D., Borgdorff, M. W., Behr, M. A., Beyers, N., van Helden, P. Proportion of tuberculosis transmission that takes place in households in a high-incidence area. Lancet 363, 212-214 (2004).

21. Bloom, B. R. \& Murray, C. J. L. Tuberculosis : Commentary on a Reemergent Killer Science. 257, 1055-1064 (1992).

22. Dormans, J., Burger, M., Aguilar, D., Hernandez-Pando, R., Kremer, K., Roholl, P., Arend, S. M., Van Soolingen, D. Correlation of virulence, lung pathology, bacterial load and delayed type hypersensitivity responses after infection with different Mycobacterium tuberculosis genotypes in a BALB/c mouse model. Clin. Exp. Immunol. 137, 460-468 (2004).

23. Petruccioli, E., Scriba, T. J., Petrone, L., Hatherill, M., Cirillo, D. M., Joosten, S. A., Ottenhoff, T. H., Denkinger, C. M., Goletti, D. Correlates of tuberculosis risk: Predictive biomarkers for progression to active tuberculosis. Eur. Respir. J. 48, 1751-1763 (2016).

24. Getahun, H., Matteelli, A., Chaisson, R. E. \& Raviglione, M. Latent Mycobacterium tuberculosis Infection. N. Engl. J. Med. 372, 2127-2135 (2015). 
25. Stallings, C. L., Glickman, M. S. Is Mycobacterium tuberculosis stressed out? A critical assessment of the genetic evidence. Microbes Infect. 12, 1091-1101 (2010).

26. Zumla, A., Raviglione, M., Hafner, R. Fordham von Reyn, C. Tuberculosis. N. Engl. J. Med. 368, 745-755 (2013).

27. Dannenberg, A. M. J. Pathogenesis of human pulmonary tuberculosis: insights from the rabbit model. (ASM Press, 2006).

28. Urbanowski, M. E., Ihms, E. A., Bigelow, K., Kübler, A., Elkington, P. T., Bishai, W. R. Repetitive Aerosol Exposure Promotes Cavitary Tuberculosis and Enables Screening for Targeted Inhibitors of Extensive Lung Destruction. J. Infect. Dis. 218, 53-63 (2018).

29. Patterson, B., Morrow, C., Singh, V., Moosa, A., Gqada, M., Woodward, J., Mizrahi, V., Bryden, W., Call, C., Patel, S., Warner, D., Wood, R. Detection of Mycobacterium tuberculosis bacilli in bio-aerosols from untreated TB patients. Gates Open Res. 1, 11 (2018).

30. Matuka, O., Singh, T. S., Bryce, E., Yassi, A., Kgasha, O., Zungu, M., Kyaw, K., Malotle, M., Renton, K., O'Hara, L. Pilot study to detect airborne Mycobacterium tuberculosis exposure in a South African public healthcare facility outpatient clinic. J. Hosp. Infect. 89, 192-196 (2015).

31. Wan, G. H., Lu, S. C., Tsai, Y. H. Polymerase chain reaction used for the detection of airborne Mycobacterium tuberculosis in health care settings. Am. J. Infect. Control 32, 1722 (2004).

32. Mostorides, S. M., Ochler, R. L., Grecne, J. N., Sinnott IV, J. T., Kranik, M., Samlin, R. L. The detection of airborne Mycobacterium tuberculosis using micropore membrane air sampling and polymerase chain reaction. Chest 115, 19-25 (1999).

33. Williams, C. M. L., Abdulwhhab, M., Birring, S., De Kock, E., Garton, N., Stoltz, A., Haldar, P., Barer, M. Twenty-four hour face mask sampling in pulmonary tuberculosis reveals three distinct patterns of bacterial aerosol production dissociated from conventional markers of transmission risk. bioRxiv 426825 (2018).

34. Wurie, F. B., Lawn, S. D., Booth, H., Sonnenberg, P., Hayward, A. C. Bioaerosol production by patients with tuberculosis during normal tidal breathing: Implications for transmission risk. Thorax 71, 549-554 (2016).

35. Lee, S., Chung, H., Park, E. Characteristics of bacteria in the living room and bathroom of a residential environment using the pyrosequencing method. Microbiol. Biotechnol. Lett. 44, 84-88 (2016).

36. Williams, C. M. L., Cheah, E. S. G., Malkin, J., Patel, H., Otu, J., Mlaga, K., Sutherland, J. S., Antonio, M., Perera, N., Woltmann, G., Haldar, P., Barer, M. R. Face mask sampling for the detection of Mycobacterium tuberculosis in expelled aerosols. PLoS One 9, 1-7 (2014).

37. Wood, R., Morrow, C., Barry, C. E., Bryden, W. A., Call, C. J., Hickey, A. J., Rodes, C. E., Scriba, T. J., Blackburn, J., Issarow, C., Mulder, N., Woodward, J., Moosa, A., Singh, V., Mizrahi, V., Warner, D. F. Real-time investigation of tuberculosis transmission: Developing the respiratory aerosol sampling chamber (RASC). PLoS One 11, 1-16 (2016).

38. Turner, R. D., Chiu, C., Churchyard, G. J., Esmail, H., Lewinsohn, D. M., Gandhi, N. R., Fennelly, K. P. Tuberculosis Infectiousness and Host Susceptibility. J. Infect. Dis. 216, S636-S643 (2017).

39. Kamariza, M., Shieh, P., Ealand, C. S., Peters, J. S., Chu, B., Rodriguez-Rivera, F. P., Babu Sait, M. R., Treuren, W. V., Martinson, N., Kalscheuer, R., Kana, B., Bertozzi, C. R. Rapid detection of Mycobacterium tuberculosis in sputum with a solvatochromic trehalose probe. Sci. Transl. Med. 10, eaam6310 (2018).

40. Rodriguez-Rivera, F. P., Zhou, X., Theriot, J. A., Bertozzi, C. R. Visualization of mycobacterial membrane dynamics in live cells. J. Am. Chem. Soc. 139, 3488-3495 (2017).

41. Kamariza, M., Shieh, P., Bertozzi, C. R. Imaging Mycobacterial Trehalose Glycolipids. Methods Enzymol. 598, 355-369 (2018).

42. Chengalroyen, M. D., Beukes, G. M., Gordhan, B. G., Streicher, E. M., Churchyard, G., Hafner, R., Warren, R., Otwombe, K, Martinson, N., Kana, B. D. Detection and quantification of differentially culturable tubercle bacteria in sputum from patients with tuberculosis. Am. J. Respir. Crit. Care Med. 194, 1532-1540 (2016). 
43. Nimmo, C., Shaw, L. P., Doyle, R., Williams, R., Brien, K., Burgess, C., Breuer, J., Balloux, F., Pym, A. S. Whole genome sequencing Mycobacterium tuberculosis directly from sputum identifies more genetic diversity than sequencing from culture. BMC Genomics $\mathbf{2 0}$, 1-9 (2019)

44. Watkins, D. A., Chahine, A., Creger, R. J., Jacobs, M. R., Lazarus, H. M. Corynebacterium striatum: A Diphtheroid with Pathogenic Potential. Clin. Infect. Dis. 17, 21-25 (1993).

45. Renom, F., Garau, M., Rubí, M., Ramis, F., Galmés, A., Soriano, J. B. Nosocomial outbreak of Corynebacterium striatum infection in patients with chronic obstructive pulmonary disease. J. Clin. Microbiol. 45, 2064-2067 (2007).

46. Alibi, S., Ferjani, A., Boukadida, J., Cano, M. E., Fernández-Martínez, M., MartínezMartínez, L., \& Navas, J. Occurrence of Corynebacterium striatum as an emerging antibiotic-resistant nosocomial pathogen in a Tunisian hospital. Sci. Rep. 7, 1-8 (2017).

47. Shaw, J., Wynn-Williams, N. Infectivity of pulmonary tuberculosis in relation to sputum status. Am. Rev. Tuberc. 69, 724-732 (1954).

48. Grzybowski, S., Barnett, G., Styblo, K. Contacts of cases of active pulmonary tuberculosis. Bull Int Union Tuberc 50, 90-106 (1975).

49. Donald, P. R., Diacon, A. H., Lange, C., Demers, A. M., von Groote-Biddlingmeier, F., Nardell, E. Droplets, dust and guinea pigs: an historical review of tuberculosis transmission research, 1878-1940. Int. J. Tuberc. Lung Dis. 22, 972-982 (2018).

50. Cheng, Y., Xie, J., Lee, K. H., Gaur, R., Song, A., Dai, T., Ren, H., Wu, J., Zhaogang, S., Banaei, N., Akin, D., Rao, J. Rapid and specific labeling of single live Mycobacterium tuberculosis with a dual-targeting fluorogenic probe. Sci. Transl. Med. 10, 1-13 (2018).

51. Sommer, R., Neres, J., Piton, J., Dhar, N., Van Der Sar, A., Mukherjee, R., Laroche, T., Dyson, P. J., McKinney, J. D., Bitter, W., Makarov, V., Cole, S. T. Fluorescent Benzothiazinone Analogues Efficiently and Selectively Label Dpre1 in Mycobacteria and Actinobacteria. ACS Chem. Biol. 13, 3184-3192 (2018).

52. Hodges, H. L., Brown, R. A., Crooks, J. A., Weibel, D. B., Kiessling, L. L. Imaging mycobacterial growth and division with a fluorogenic probe. Proc. Natl. Acad. Sci. 115, 5271-5276 (2018).

53. Borgdorff, M. W., van Soolingen, D. The re-emergence of tuberculosis: What have we learnt from molecular epidemiology? Clin. Microbiol. Infect. 19, 889-901 (2013).

54. Andrews, J. R., Morrow, C., Walensky, R. P., Wood, R. Integrating social contact and environmental data in evaluating tuberculosis transmission in a South African township. $J$. Infect. Dis. 210, 597-603 (2014).

55. Martinez, L., le Roux, D. M., Barnett, W., Stadler, A., Nicol, M. P., Zar, H. J. Tuberculin skin test conversion and primary progressive tuberculosis disease in the first 5 years of life: a birth cohort study from Cape Town, South Africa. Lancet Child Adolesc. Heal. 2, 46-55 (2018).

56. Martinez, L., Shen, Y., Mupere, E., Kizza, A., Hill, P. C., Whalen, C. C. Transmission of Mycobacterium Tuberculosis in Households and the Community: A Systematic Review and Meta-Analysis. Am. J. Epidemiol. 185, 1327-1339 (2017).

57. Corbett, E. L., Bandason, T., Cheung, Y. B., Makamure, B., Dauya, E., Munyati, S. S., Churchyard, G. J., Williams, B. G., Butterworth, A. E., Mungofa, S., Hayes, R. J., Mason, P. R. Prevalent infectious tuberculosis in Harare, Zimbabwe: Burden, risk factors and implications for control. Int. J. Tuberc. Lung Dis. 13, 1231-1237 (2009).

58. Horton, K. C., Sumner, T., Houben, R. M. G. J., Corbett, E. L., White, R. G. A Bayesian Approach to Understanding Sex Differences in Tuberculosis Disease Burden. Am. J. Epidemiol. 187, 2431-2438 (2018).

59. Wood, R., Middelkoop, K., Myer, L., Grant, A. D., Whitelaw, A., Lawn, S. D., Kaplan, G., Huebner, R., McIntyre, J., Bekker, L. G. Undiagnosed tuberculosis in a community with high HIV prevalence: Implications for tuberculosis control. Am. J. Respir. Crit. Care Med. 175, 87-93 (2007).

60. Shah, N. S., Auld, S. C., Brust, J. C. M., Mathema, B., Ismail, N., Moodley, P., Mlisana, K., Allana, S., Campbell, A., Mthiyane, T., Morris, N., Mpangase, P., van der Meulen, H., Omar, S. V., Brown, T. S., Narechania, A., Shaskina, E., Kapwata, T., Kreiswirth, B., 
Gandhi, N. R. Transmission of extensively drug-resistant tuberculosis in South Africa. N. Engl. J. Med. 376, 243-253 (2017).

61. Drain, P. K., Bajema, K. L., Dowdy, D., Dheda, K., Naidoo, K., Schumacher, S. G., Ma, S., Meermeier, E., Lewinsohn, D M., Sherman, D. R. Incipient and subclinical tuberculosis: A clinical review of early stages and progression of infection. Clin. Microbiol. Rev. 31, 1-24 (2018).

62. Ducret, A., Quardokus, E., Brun, Y. MicrobeJ, a tool for high throughput bacterial cell detection and quantitative analysis. Nat. Microbiol. 1, 1-14 (2016).

Acknowledgments: We are grateful to Lizette Koekemoer for technical assistance in the preparation of starved $M$. tuberculosis H37Rv cultures. Funding: The authors acknowledge the financial support of the South African Medical Research Council (SAMRC) with funds from National Treasury under its Economic Competitiveness and Support Package (MRC-RFA-UFSP01-2013/CCAMP, RW), the Strategic Health Innovations Partnerships (SHIP) Unit of the SAMRC (to D.F.W and V.M) and as a sub-grant from the Bill and Melinda Gates Foundation (R.W.), and the SAMRC extramural unit funding (to V.M.). We are grateful to the Bill \& Melinda Gates Foundation (OPP1116641, RW), the Research Council of Norway (R\&D Project 261669 "Reversing antimicrobial resistance", D.F.W.), and the US National Institute of Child Health and Human Development (NICHD) U01HD085531 (D.F.W.). We also acknowledge the funding support of the National Research Foundation of South Africa (V.M.) and the Howard Hughes Medical Institute for a Senior International Research Scholars grant (V.M.). We acknowledge support by Stanford University's Diversifying Academia, Recruiting Excellence Fellowship, and the NIH Predoctoral Fellowship F31AI129359 (M.K). Lastly, we acknowledge funding from the Bill and Melinda Gates Foundation (OPP115061) and NIH (AI051622) grants (to C.R.B.). Author contributions: R.D., S.G., R.W. and D.F.W. designed and led the study. R.W. directed the clinical research site, and C.M. and M.G. assisted with the recruitment of TB patients, ethics application and approval, and transport of the bioaerosol and sputum samples. M.K. and C.R.B provided DMN-trehalose (-tre) reagent and technical input into mycobacterial staining. R.D. performed the BSL2 work and the microscopy. S.G. performed BSL3 experiments, including bioaerosol and sputum sample processing and DMN-tre staining. The nanowell arrayed slides were synthesized by B.S., C.Mc. and A.K., and designed in conjunction with R.D., S.G., and D.F.W. Technical oversite of the RASC was provided by R.W., W.B. and C.C. Data were analyzed by R.D., S.G., and D.F.W. who wrote and edited the manuscript which was approved by all authors.. Competing interests: C.R.B. and M.K. are cofounders of OliLux Biosciences. CRB is a member of the Board of Directors of Lilly and a cofounder of Enable Biosciences, Palleon Pharmaceuticals, and InterVenn Bio. 